\title{
Simulación numérica de estructura metálica ensayada en mesa vibradora
}

\author{
Francisco López Almansa ${ }^{1}$, Gustavo Palazzo ${ }^{2}$, Francisco Calderón ${ }^{2}$, Victor Roldán ${ }^{2}$
}

\section{RESUMEN}

En el laboratorio E-Defense de Japón se ensayó durante 2007 un prototipo de edificio, a escala real, cuya estructura estaba conformada por pórticos metálicos resistentes a momentos. Ese prototipo fue sometido a diferentes escalas de las tres componentes del acelerograma registrado en la estación de Takatori, durante el terremoto de Kobe de 1995. El objetivo del presente trabajo es evaluar la capacidad de modelos computacionales para reproducir la respuesta dinámica no lineal del prototipo. Para ello se hace primero una descripción del prototipo de ensayo y de los modelos computacionales considerados y, posteriormente, se desarrolla un estudio de los resultados. Así, se comparan valores máximos de respuesta numéricos y experimentales, y también las historias de desplazamientos y aceleraciones en el último nivel de los modelos. En las conclusiones se destacan las características principales de cada simulación, y se plantean aspectos fundamentales a considerar en un modelo numérico para lograr reproducir un comportamiento experimental de una estructura metálica en campo no lineal.

Palabras claves: estructura metálica sismorresistente, análisis dinámico no lineal, resultados experimentales vs. numérico.

1 Universidad Politécnica de Cataluña, Departamento de Estructuras en Arquitectura, Avda. Diagonal 649, 08028 Barcelona, España, francesc.lopez-almansa@upc.edu

2 Universidad Tecnológica Nacional, Facultad Regional Mendoza, Ceredetec, Rodríguez 273, 5500 Ciudad de Mendoza, Argentina, gpalazzo@frm.utn.edu.ar, francisco.calderon@frm.utn.edu.ar, victor.roldan@frm.utn.edu.ar

http://dx.doi.org/10.5335/rsee.v110i1 


\section{Introducción}

En este trabajo se ha seleccionado como objeto de estudio el prototipo que se presenta en la Figura 1. El mismo corresponde a una estructura de acero de cuatro niveles, conformada por un pórtico espacial resistente a momentos, y ensayado durante 2007 en la mesa vibradora del Laboratorio E-Defense (Japón).
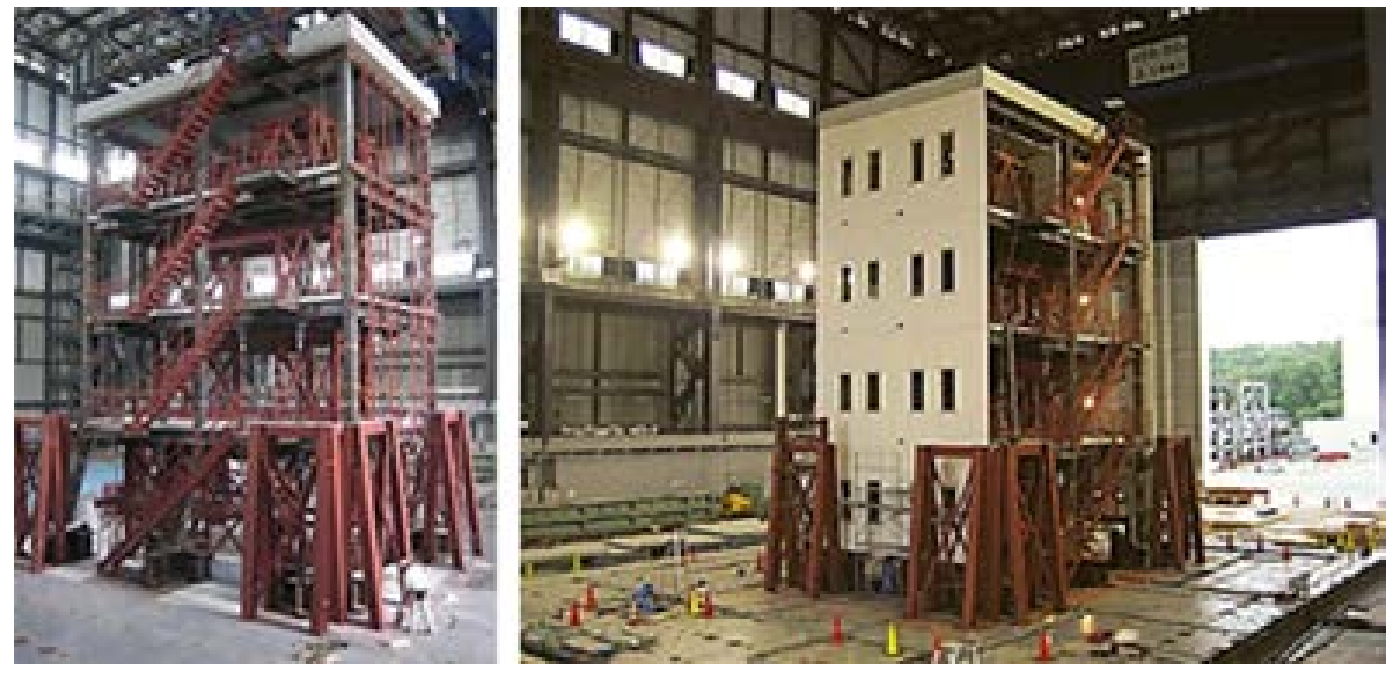

Figura 1: Pórtico espacial de acero, ensayado en el E-Defense en 2007.

La estructura fue sometida a las tres componentes del acelerograma de Kobe de 1995, registrado en la estación Takatori. Cada componente se aplicó escalada, en registros sucesivos, al 40, 60 y $100 \%$.

Desde el E-Defense se organizó un concurso ciego, por lo que fueron provistos todos los datos geométricos y mecánicos de la estructura antes de la realización del ensayo; así como los parámetros de respuesta una vez realizado el mismo.

A los efectos de determinar el grado de aproximación que puede lograrse en una simulación computacional para reproducir la respuesta dinámica no lineal del prototipo sometido a las 3 componentes del acelerograma mencionado, se desarrolla el presente trabajo.

Se consideran para ello los siguientes modelos computacionales: i- Modelo I: Modelo descripto en Pavan (2008); ii- Modelo II: Modelo del prototipo incluido en los Ejemplos de Verificación del programa SeismoStruct (2013a), y descripto también en el Informe de Verificación de ese software (SeismoStruct, 2013b), basado en datos de Pavan (2008); iii- Modelo III: Realizado por los autores del presente trabajo, tomando como base los datos indicados en Pavan (2008), pero incorporando ciertas consideraciones no incluidas en el primer modelo. Fue necesario considerar el Modelo II de Pavan (2008) 
para poder realizar comparaciones de las historias de respuesta. Además, este modelo se lo diferencia del Modelo I, ya que el primero es parte de los ejemplos de verificación de SeismoStruct (2013a), y el segundo está descripto en Pavan (2008).

$\mathrm{El}$ análisis estructural de los tres modelos se realizó con el programa de elementos finitos SeismoStruct (2013a), capaz de predecir el comportamiento de pórticos espaciales bajo excitación sísmica, considerando no linealidades geométricas y del material.

El trabajo se divide en cinco secciones: i- en la primera se presentan los datos del prototipo; ii- en la segunda, tercera y cuarta se explican las consideraciones realizadas para construir los Modelo I, II y III respectivamente; iii- en la cuarta sección se comparan resultados numéricos y experimentales, así como los resultados numéricos entre sí; y iv) en la última sección se presentan las conclusiones.

\section{Caracterización del prototipo}

Los datos correspondientes al prototipo fueron obtenidos de Pavan (2008), Ohsaki et al. (2008), Tada et al. (2007), y Yamada et al. (2008).

\subsection{Geometría}

El prototipo consiste en dos pórticos de acero con dos vanos de $5.0 \mathrm{~m}$ de longitud (dirección $\mathrm{X}$ ), y tres pórticos de acero con un vano de $6.0 \mathrm{~m}$ de longitud (dirección $\mathrm{Y}$ ). En la Tabla 1 se presenta la altura para cada piso y nivel, mientras que en las Figuras 2 se muestran los parámetros que definen la geometría general de la estructura.

Tabla 1: Altura en [m] de pisos y niveles en el prototipo.

\begin{tabular}{c|c|c|c}
\hline Piso & Altura respecto a la sup. de la mesa & Nivel & Altura del nivel \\
\hline Parapeto & 16.475 & Parapeto & 0.600 \\
Techo & 15.875 & 4 & 3.500 \\
4to & 12.375 & 3 & 3.500 \\
3ro & 8.875 & 2 & 3.500 \\
2do & 5.375 & 1 & 3.875 \\
1ro & 1.500 & \multicolumn{2}{|c}{-} \\
\hline
\end{tabular}




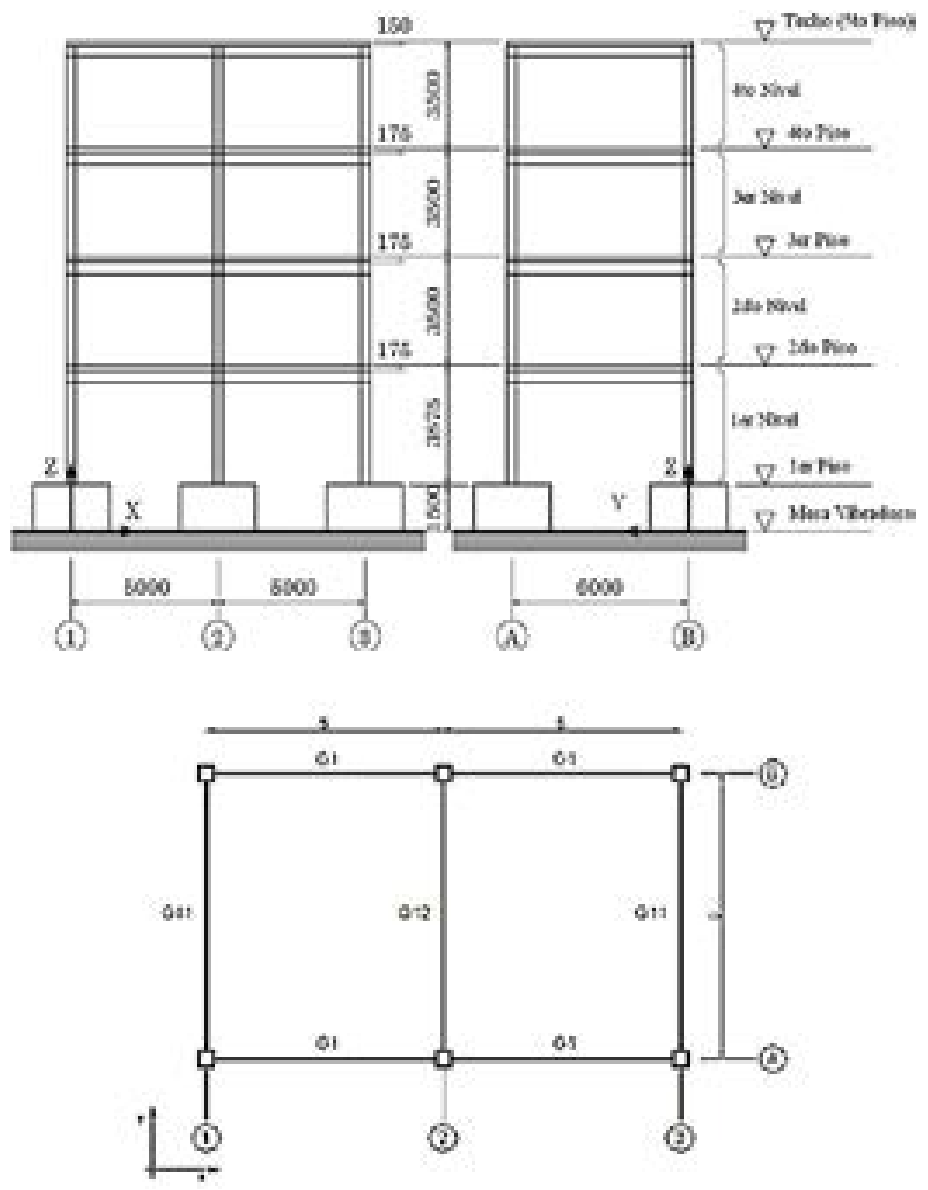

Figura 2: Parámetros geométricos del prototipo en [mm].

Los pórticos estaban conformados por columnas tubulares cuadradas y vigas de alas anchas. En las Tablas 2 y 3 se presentan las dimensiones de estos elementos estructurales.

Tabla 2: Dimensiones de columnas y vigas principales en [mm].

\begin{tabular}{|c|c|c|c|c|c|}
\hline \multicolumn{4}{|c|}{$\operatorname{Vigas}^{(1)}$} & \multicolumn{2}{|c|}{ Columnas $^{(2)}$} \\
\hline Piso & G1 & G11 & G12 & Nivel & - \\
\hline 5 & $H-346_{x} 174_{x} 6 x_{x} 9$ & $H-346_{x} 174_{x} 66_{x} 9$ & $H-346_{x} 174_{x} 6_{x} 9$ & 4 & RHS $-300 x 300_{x} 9$ \\
\hline 4 & $H-350 x 175_{x} 7_{x} 11$ & $H-350 x 175_{x} 7 x 11$ & $H-340 x 175_{x} 9_{x} 14$ & 3 & RHS - $300 x 300 x 9$ \\
\hline 3 & $H-396_{x} 199_{x} 7_{x} 11$ & $H-400 x 200 x 8_{x} 13$ & $H-400 x 200 x 8_{x} 113$ & 2 & RHS $-300 x 300 x 9$ \\
\hline 2 & $H-400_{x} 200_{x} 8_{x} 13$ & $H-400 x 200 x 8_{x} 13$ & $H-390_{x} 200_{x} 10_{x} 16$ & 1 & RHS $-300 x 300_{x} 9$ \\
\hline
\end{tabular}

(1) $\mathrm{H}$ - altura ${ }_{x}$ ancho ${ }_{x}$ espesor del alma ${ }_{x}$ espesor de las alas

(2) RHS - altura ${ }_{x}$ ancho ${ }_{x}$ espesor 
Tabla 3: Dimensiones vigas secundarias en [mm].

\begin{tabular}{c|c}
\hline B20 & $H-200_{x} 100_{x} 5.5_{x} 8$ \\
B29 & $H-294_{x} 200_{x} 8_{x} 12$ \\
B34 & $H-346_{x} 174_{x} 69$ \\
B35 & $H-350_{x} 175_{x} 7_{x} 11$ \\
\hline
\end{tabular}

\subsection{Losas}

Las losas de hormigón con sistema steel deck, para el segundo, tercer y cuarto piso, tenían un espesor de $175 \mathrm{~mm}$, como se observa en la Figura 3, a); mientras que la losa de techo, de $150 \mathrm{~mm}$ de espesor, era de hormigón armado (ver Figura 3, b).

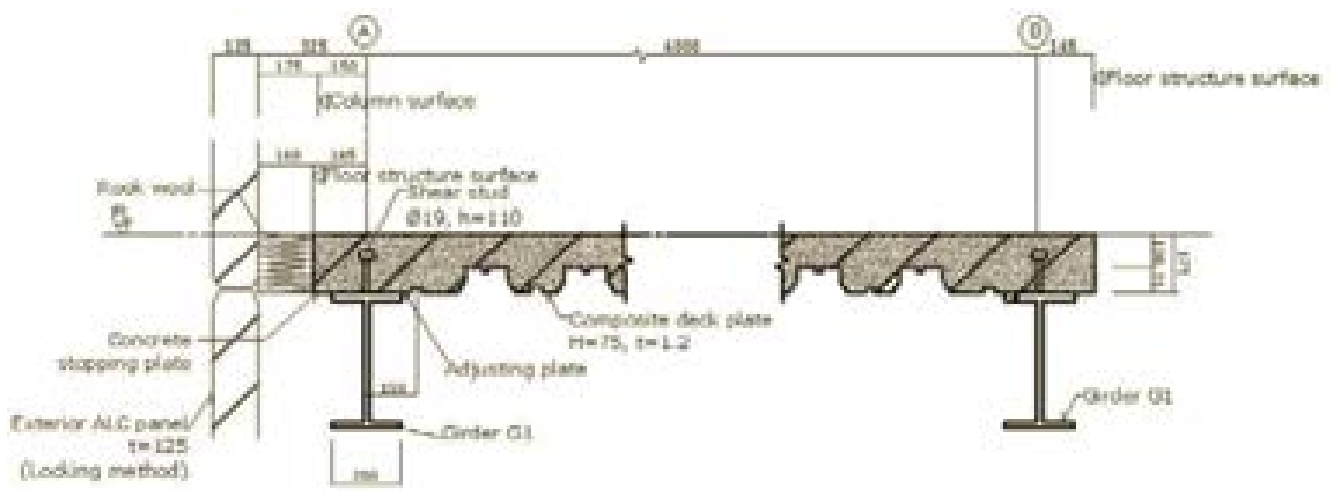

a) Losas pisos 2, 3 y 4

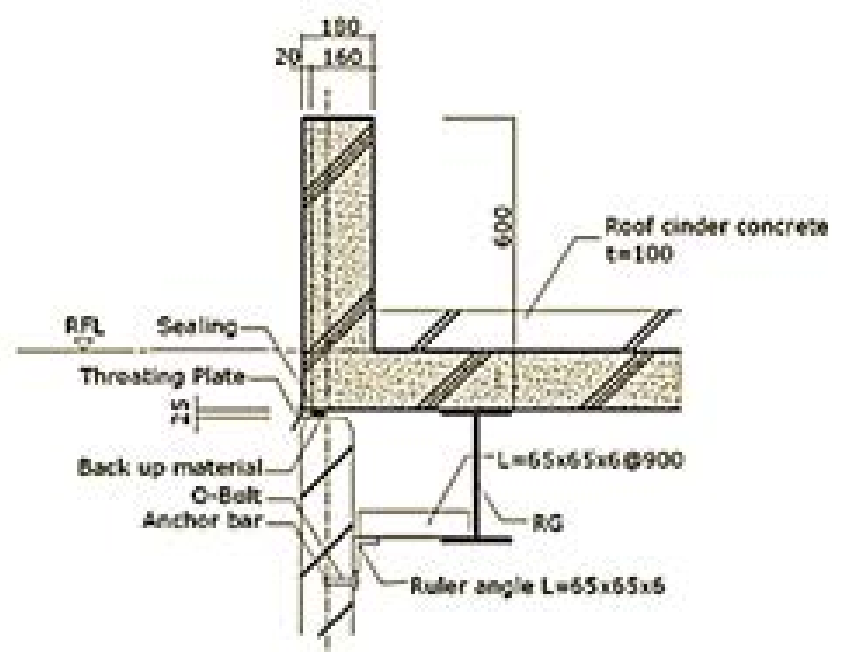

b) Losa de techo

Figura 3: Sección transversal de losas. 
En la Tabla 4 se presentan los parámetros del hormigón de las losas medidos durante el ensayo (resistencia a compresión $\mathrm{f}_{\text {cu }}$, resistencia a tracción $\mathrm{f}_{\mathrm{t}}$, deformación para el valor máximo de tensión en el hormigón $\varepsilon_{\mathrm{c}}$ ).

Tabla 4: Parámetros del hormigón en losas.

\begin{tabular}{c|c|c|c}
\hline Piso & $\mathrm{f}_{\text {cu }}[\mathrm{MPa}]$ & $\mathrm{f}_{\mathrm{t}}[\mathrm{MPa}]$ & $\varepsilon_{\mathrm{c}}$ \\
\hline 2do & 33.53 & 3.35 & 0.00154 \\
3ro & 28.80 & 2.88 & 0.00120 \\
4to & 27.54 & 2.75 & 0.00127 \\
Techo & 35.90 & 3.59 & 0.00247 \\
\hline
\end{tabular}

\subsection{Conexiones}

Todas las conexiones fueron realizadas según detalles y prácticas de fabricación desarrolladas de acuerdo a las enseñanzas del terremoto de Kobe de 1995. La Figura 4 muestra una conexión típica viga - columna. Primero se construyó un "árbol columna", donde sectores de vigas se sueldan al mismo. Luego el resto de las vigas se conectaron al "árbol" mediante conexiones abulonadas. Este tipo de conexiones fuerza la formación eventual de rótulas plásticas fuera del punto más débil de la conexión.
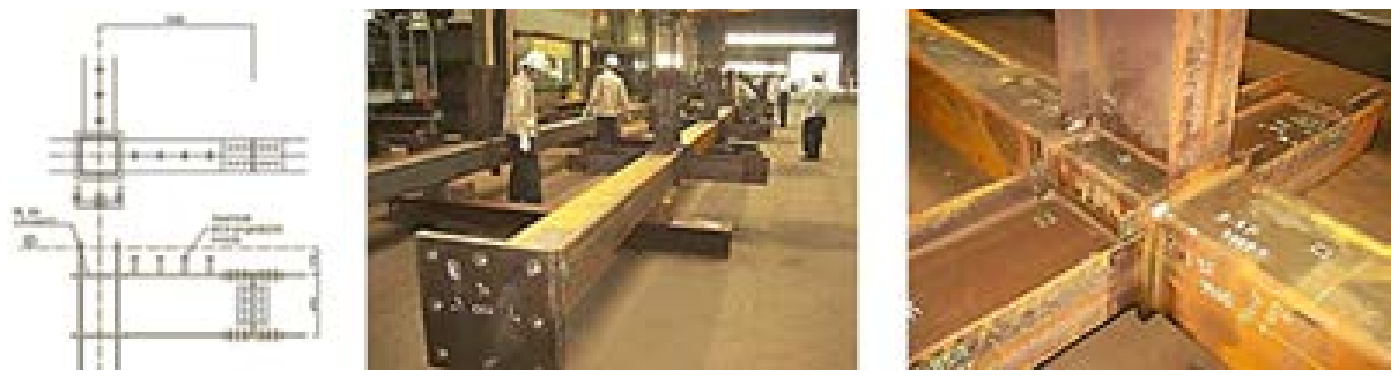

Figura 4: Conexión viga - columna. 


\subsection{Elementos no estructurales}

\subsubsection{Paredes externas}

Las paredes externas del prototipo estaban conformadas de hormigón aireado, de $0,125 \mathrm{~m}$ de espesor.

Estos paneles tenían conexiones que permitían el cabeceo de los paneles en el caso de un terremoto. Esas conexiones a las vigas principales estaban materializadas por ángulos y placas, a los efectos de mantener el equilibrio y evitar el vuelco ante acciones sísmicas. Pero los paneles eran efectivamente soportados por perfiles de ala ancha ubicados en la parte inferior del edificio. De esta manera, el peso de los mismos era descargado directamente sobre sus vigas de fundación, sin afectar otros elementos estructurales.

\subsubsection{Particiones internas, aberturas y cielorrasos}

Como particiones internas se emplearon placas, instaladas sobre marcos de aluminio. También se incorporaron ventanas con marcos de aluminio en los paneles externos. Se usaron además barras de acero de bajo peso para sostener placas de cielo raso. En la Figura 5 se presentan algunas de las componentes mencionadas.
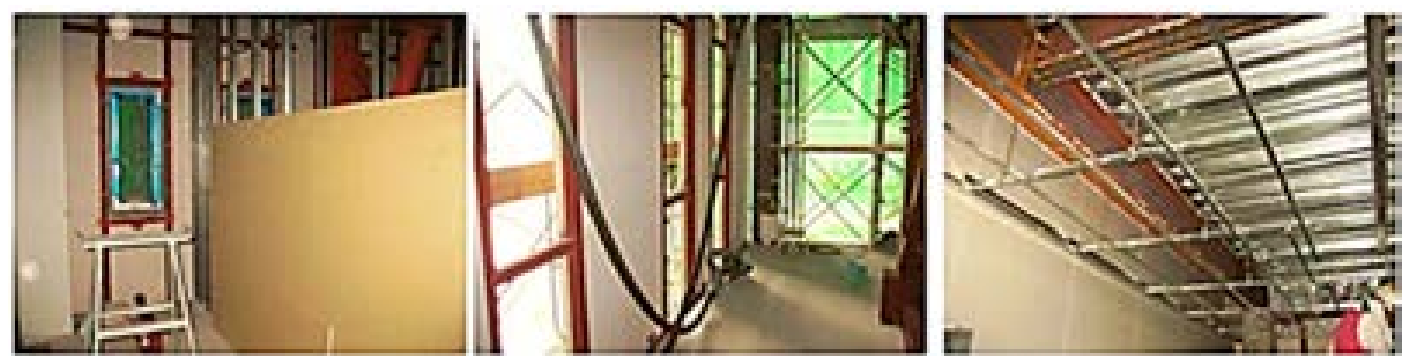

Figura 5: Particiones internas, ventanas y barras soporte de cielo raso.

\subsection{Pesos}

Los pesos de cada parte de la estructura, según información suministrada por los organizadores del concurso, se presentan en la Tabla 5. 
Tabla 5: Pesos en $[\mathrm{kN}]$ parciales.

\begin{tabular}{c|c|c|c|c|c|c|c|c|c}
\hline & Piso & Pórtico & Muros exter. & Muros int. & Cielorraso & Parapeto & $\begin{array}{c}\text { Sistema } \\
\text { de seg. }\end{array}$ & $\begin{array}{c}\text { Piso } \\
\text { vol. }\end{array}$ & Total \\
\hline Techo & 458 & 21 & 0 & 0 & 13 & 71 & 0 & 2 & 565 \\
4to P. & 0 & 19 & 79 & 35 & 0 & 0 & 0 & 0 & 133 \\
4to P. & 270 & 24 & 0 & 0 & 3 & 0 & 48 & 4 & 349 \\
3er P. & 0 & 18 & 74 & 30 & 0 & 0 & 0 & 0 & 122 \\
3er P. & 260 & 32 & 0 & 0 & 3 & 0 & 47 & 5 & 347 \\
2do P. & 0 & 18 & 73 & 30 & 0 & 0 & 9 & 0 & 130 \\
2do P. & 260 & 41 & 0 & 0 & 0 & 0 & 47 & 4 & 352 \\
1er P. & 0 & 27 & 76 & 0 & 0 & 0 & 12 & 0 & 115 \\
Total & 1248 & 200 & 302 & 95 & 19 & 71 & 163 & 15 & 2113 \\
\hline
\end{tabular}

Cada parte incluye: Piso: losa, vigas, barandas. Pórtico (primera fila): columnas, diafragmas y paneles de conexión. Pórtico (segunda fila): bulones, materiales resistentes al fuego. Muros exteriores: paneles, ventanas y sus marcos. Muros interiores: placas y soportes, puertas. Cielorrasos: placas y sistema soporte. Parapeto: viga de borde de hormigón armado a nivel de techo. Sistema de seguridad: elementos metálicos. Piso en voladizos: piso para soporte transitorio de escalera y barandas.

En la Tabla 6 se indican los pesos totales considerados para cada piso. No se consideró el peso de los muros exteriores, ya que estos apoyaban en una viga en el nivel inferior.

Tabla 6: Pesos totales en [kN] por piso.

\begin{tabular}{c|c}
\hline Piso & Peso por piso \\
\hline 5 & 631.5 \\
4 & 476.5 \\
3 & 473.0 \\
2 & 474.5 \\
Total & 2055.5 \\
\hline
\end{tabular}

\subsection{Excitación en la base}

El prototipo fue sometido a una excitación dinámica en la base hasta el colapso, aplicando distintas escalas de los acelerogramas para la estación de Takatori, durante el terremoto de Kobe de 1995. Se consideraron las componentes norte - sur, este oeste y vertical de la estación mencionada. Estas componentes se aplicaron escaladas al 40\% (nivel elástico), al 60\% (nivel sísmico de colapso incipiente, comportamiento elasto-plástico), y al 100\% (nivel sísmico de colapso). Para este trabajo fueron seleccionadas solo las dos primeras escalas, ya que los parámetros de respuesta experimental se determinaron para este nivel de excitación. La componente norte - sur de los acelerogramas fue aplicada en la dirección longitudinal del prototipo (dirección X), y la componente en la dirección este - oeste en la dirección transversal (dirección Y). En la 
Figura 6 se muestran los registros medidos en la base durante el ensayo dinámico; sin considerar los registros con escala del $100 \%$ por las razones antes expuestas.
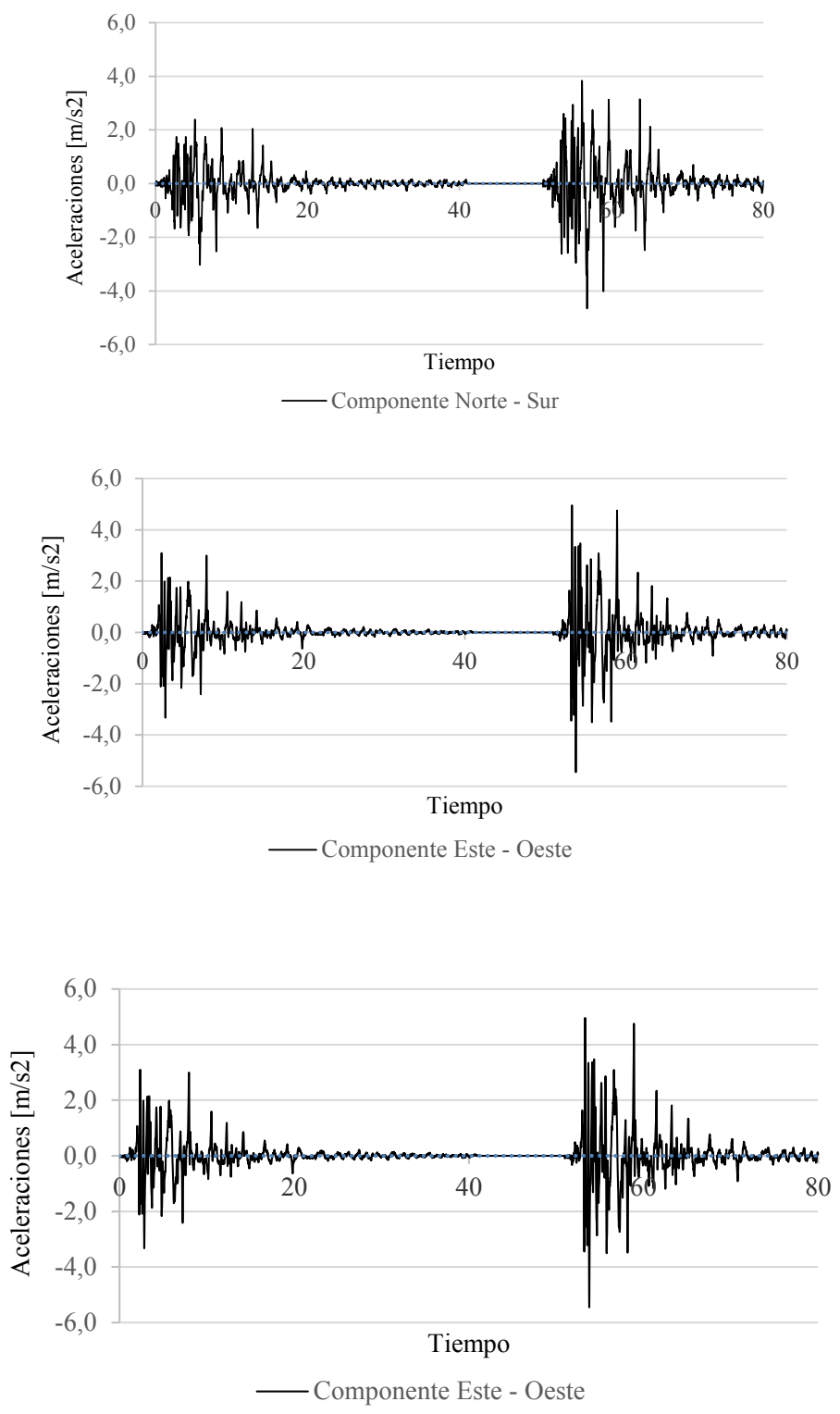

Figura 6: Excitación sísmica en la base (escala del 40 y 60\%).

Los valores pico de aceleración (PGA) de cada registro, para las diferentes escalas, se presentan en Tabla 7. 
Tabla 7: Valores pico de PGA en $\left[\mathrm{m} / \mathrm{s}^{2}\right]$ para diferentes intensidades de la excitación.

\begin{tabular}{c|l|l|l}
\hline Intensidad & $40 \%$ & $60 \%$ & $100 \%$ \\
\cline { 2 - 4 } Componente & \multicolumn{3}{|c|}{ PGA } \\
\hline Norte - Sur & 3.028 & 4.658 & 8.566 \\
Este - Oeste & 3.327 & 5.451 & 8.492 \\
Vertical & 1.429 & 2.348 & 3.848 \\
\hline
\end{tabular}

Durante la aplicación de los registros en escala real, se produjo el colapso de la estructura luego de $6.2 \mathrm{~s}$ de iniciada la excitación, según se muestra la Figura 7, con formación de rótulas plásticas en cabeza y pie de columnas del primer nivel.
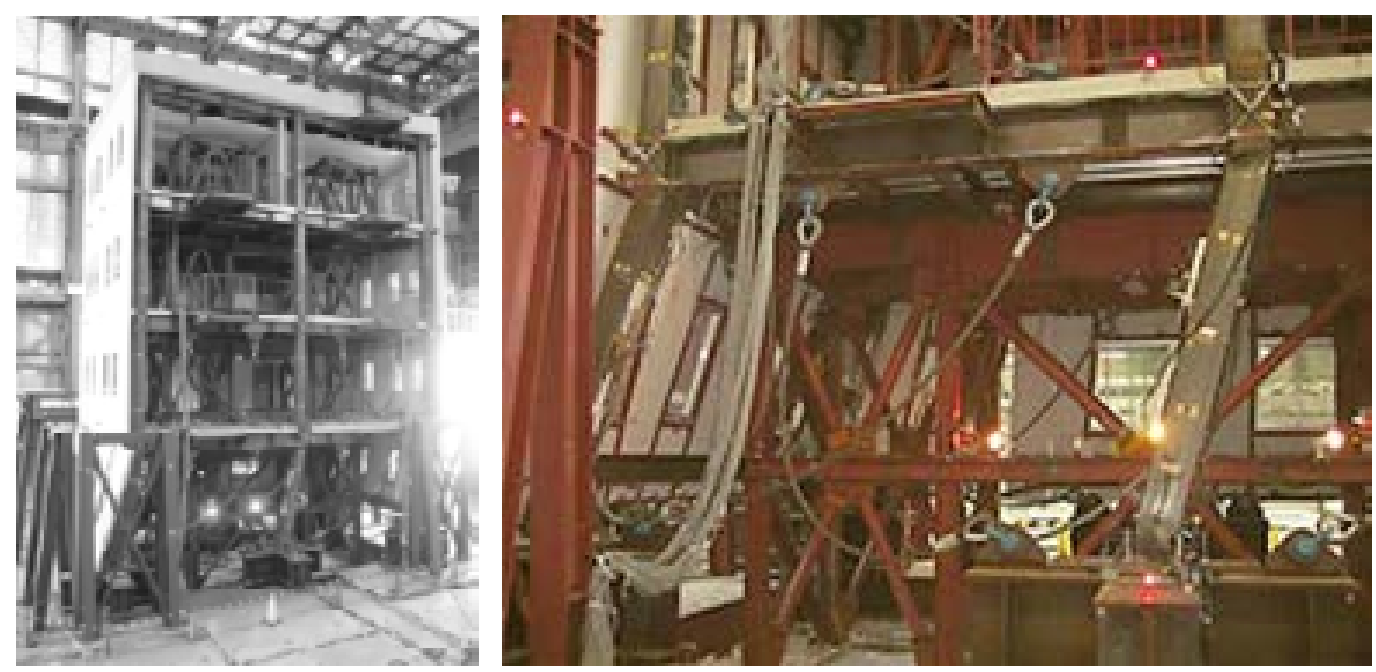

Figura 7: Estado final del prototipo (excitación a escala del 100\%).

\section{Simulación numérica de Pavan para modelo I}

Los datos que se presentan en esta sección fueron tomados de Pavan (2008).

\subsection{Geometría}

La geometría desarrollada para el prototipo se presenta en la Figura 8. 


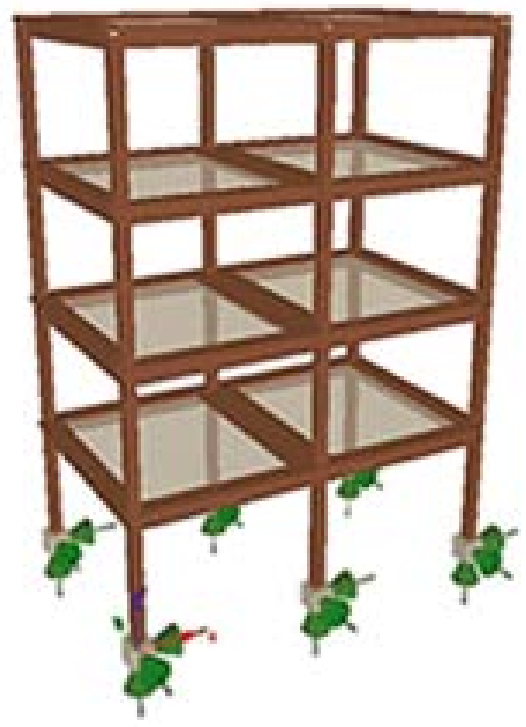

Figura 8: Geometría del Modelo I, según Pavan (2008).

El comité organizador del concurso presentó los resultados de ensayos cíclicos de una unión viga - columna y de una columna anclada en base, a los efectos de obtener relaciones constitutivas para esta parte del prototipo. Pero en el modelo de Pavan (2008) esas zonas no fueron consideradas con una simulación especial. Los elementos vigas se conectaron directamente a los nodos a donde concurrían los elementos columna. Esta simplificación se hizo bajo la hipótesis de que la contribución del panel a la deformación global de la estructura podría compensarse por la ausencia en el modelo de los elementos no estructurales (los cuales suman rigidez y reducen la deflexión estructural).

\subsection{Materiales}

Para el hormigón de las losas se consideraron los parámetros mecánicos dados en Tabla 4.

Para el acero de los distintos elementos estructurales se consideraron los parámetros dados por los organizadores del concurso, que se presentan en la Tabla 8.

Si bien fueron proporcionados los parámetros mecánicos del acero para las alas y el alma, en el modelo solo se incluyeron los parámetros mecánicos de las alas, ya que no puede ingresarse dos clases de acero para una sección. Además, se consideraron las alas, ya que representan mejor el comportamiento post fluencia por ser la primera parte de un perfil que plastifica. 
Para el acero de columna se adoptó un módulo de elasticidad E de $200000 \mathrm{MPa}$, y una tensión de fluencia $\mathrm{f}_{\mathrm{y}}$ de $380 \mathrm{MPa}$. Los valores dados para estos parámetros por los organizadores del concurso del E-Defense fueron $\mathrm{E}=90000 \mathrm{MPa} \mathrm{y} \mathrm{f}_{\mathrm{y}}=330 \mathrm{MPa}$. En Pavan (2008) se justifica el cambio, con los cuales se logra una respuesta numérica cercana a la experimental.

Tabla 8: Parámetros mecánicos para el acero de los elementos estructurales.

\begin{tabular}{|c|c|c|c|c|}
\hline Elemento & & $\mathrm{E}[\mathrm{MPa}]$ & $\mathrm{f}_{\mathrm{y}}[\mathrm{MPa}]$ & $\begin{array}{l}\text { Parámetros de } \\
\text { endurecimiento }\end{array}$ \\
\hline Columna 1 & & 200000 & 380.0 & 0.05 \\
\hline Columna 2 & & 200000 & 380.0 & 0.05 \\
\hline \multirow{2}{*}{$2 \mathrm{G} 1,2 \mathrm{G} 11,3 \mathrm{G} 11,3 \mathrm{G} 12$} & Alas & 203750 & 326.0 & 0.12 \\
\hline & Alma & 181951 & 373.0 & - \\
\hline \multirow{2}{*}{$4 \mathrm{G} 12$} & Alas & 212827 & 308.6 & 0.10 \\
\hline & Alma & 211562 & 354.5 & - \\
\hline \multirow{2}{*}{ RG1, RG11, RG12 } & Alas & 201818 & 333.0 & 0.15 \\
\hline & Alma & 206703 & 382.4 & - \\
\hline \multirow{2}{*}{$4 \mathrm{G} 1,4 \mathrm{G} 11$} & Alas & 223482 & 301.7 & 0.13 \\
\hline & Alma & 216364 & 349.9 & - \\
\hline \multirow{2}{*}{$2 \mathrm{G} 12$} & Alas & 174765 & 297.1 & 0.20 \\
\hline & Alma & 204258 & 316.6 & - \\
\hline \multirow{2}{*}{$3 \mathrm{G} 1$} & Alas & 199551 & 311.3 & 0.18 \\
\hline & Alma & 194211 & 369.0 & - \\
\hline
\end{tabular}

Desde el E-Defense no se proveyó el parámetro de endurecimiento dado en la Tabla 8, sino las leyes constitutivas para cada material obtenidas en base a ensayos de tracción. Considerando estas leyes, Pavan (2008) obtuvo los parámetros de endurecimiento de la Tabla anterior, aplicando el principio de igual disipación de energía.

El elemento "Columna 1" en la Tabla, está referido a las columnas del primer nivel, y el elemento "Columna 2" hace referencia al resto de las columnas.

\subsection{Secciones}

\subsubsection{Clase de sección considerada}

En las vigas se consideró el ancho colaborante de la losa que éstas soportaban. En la Tabla 9 se presenta dicho ancho, junto con el espesor de la losa. La determinación de los anchos colaborantes la realizó Pavan (2008), tomando como base los requerimientos de 2 reglamentos, y un estudio numérico. Según la Figura 3, el espesor total de las losas con steel deck, fue de $0.17 \mathrm{~m}$, presentando un espesor uniforme de hormigón de 0.10 m. Entonces, Pavan (2008) consideró un espesor de la losa colaborante de $0.10 \mathrm{~m}$ para las vigas en dirección $\mathrm{X}$, y de $0.1375 \mathrm{~m}$ en la dirección Y. 
Tabla 9: Ancho colaborante y espesor de losas en vigas en [m].

\begin{tabular}{c|c|c|c}
\hline \multirow{3}{*}{ Piso } & Elemento & Ancho colaborante & Espesor \\
\hline \multirow{3}{*}{5} & RG1 & 0.496 & 0.1500 \\
\cline { 2 - 4 } & RG11 & 0.496 & 0.1500 \\
\cline { 2 - 4 } & RG12 & 0.992 & 0.1500 \\
\hline \multirow{3}{*}{4} & $4 G 1$ & 0.525 & 0.1000 \\
\cline { 2 - 4 } & $4 G 11$ & 0.525 & 0.1375 \\
\hline \multirow{3}{*}{3} & $4 G 12$ & 1.050 & 0.1375 \\
\cline { 2 - 4 } & $3 G 1$ & 0,575 & 0.1000 \\
\cline { 2 - 4 } & $3 G 11$ & 0.575 & 0.1375 \\
\hline \multirow{2}{*}{2} & $3 G 12$ & 1.150 & 0.1375 \\
\cline { 2 - 4 } & $2 G 1$ & 0.575 & 0.1000 \\
\hline & $2 G 11$ & 0.575 & 0.1375 \\
\hline
\end{tabular}

\subsubsection{Masa adicional}

Para determinar la cantidad de masa adicional en cada viga, Pavan (2008) definió áreas tributarias según se muestra en la Figura 9, que en total sumaban los $60 \mathrm{~m}^{2} \mathrm{de}$ superficie de cada piso.

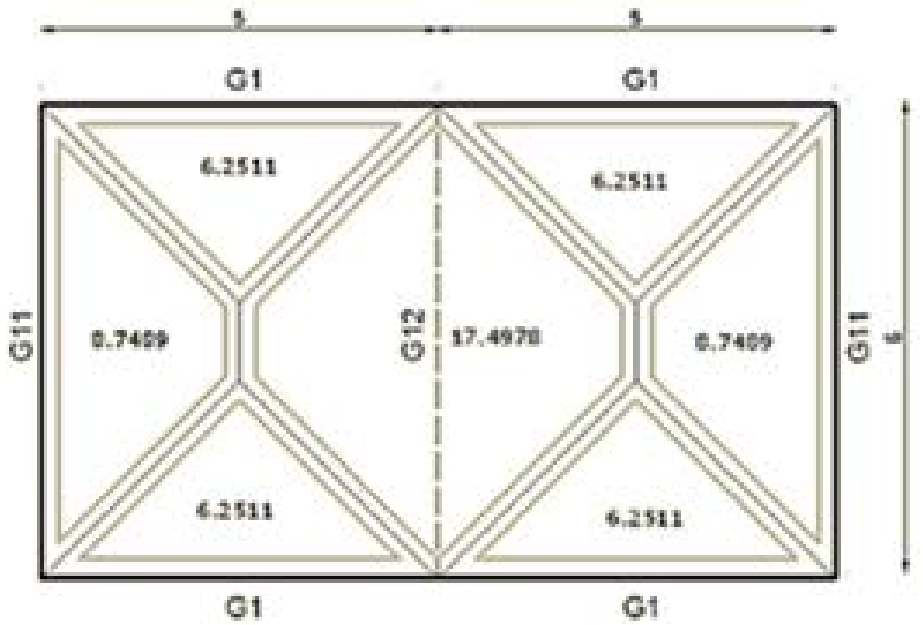

Figura 9: Áreas tributarias en $\left[\mathrm{m}^{2}\right]$ para determinar la masa adicional en vigas.

Si a partir del peso de un piso, se resta el peso propio de los elementos estructurales de acero, se tiene el peso propio de la losa de ese piso. Si a este peso se le resta el peso del ancho de losa considerado en la sección del elemento viga - losa, se tiene el peso a agregar como masa adicional en las vigas. Las masas así obtenidas son dadas en la Tabla 10. 
Tabla 10: Masa adicionales en [t / m] agregadas en vigas.

\begin{tabular}{c|c|c}
\hline Piso & Elemento & Masa adicional \\
\hline \multirow{3}{*}{5} & $R G 1$ & 1.155 \\
& $R G 11$ & 1.309 \\
4 & $R G 12$ & 2.165 \\
& $4 G 1$ & 0.757 \\
& $4 G 11$ & 0.883 \\
3 & $4 G 12$ & 1.766 \\
& $3 G 1$ & 0.728 \\
2 & $3 G 11$ & 0.849 \\
& $3 G 12$ & 1.698 \\
& $2 G 1$ & 0.724 \\
& $2 G 11$ & 0.844 \\
\hline
\end{tabular}

En las vigas RG1 y RG11 se consideró también el peso del parapeto.

Para las vigas de techo (RG1, RG11 y RG12), no se menciona en Pavan (2008) la consideración del hormigón alivianado de $0.10 \mathrm{~m}$ de espesor, colocado sobre la losa de techo (ver Figura 3).

\subsection{Excitación en la base}

En cada nodo de pie de columna se aplicaron las componentes norte - sur (en la dirección $\mathrm{X}$ ), este - oeste (en la dirección $\mathrm{Y}$ ) y vertical de los acelerogramas registrados en la estación Takatori para el terremoto de Kobe de 1995, con las escalas del 40 y 60\%, según se mencionó en la sección 2 (ver Figura 6).

\section{Simulación numérica de Pavan para modelo II}

En esta sección se hace referencia al modelo del pórtico E-Defense incluido en los Ejemplos de Verificación del programa SeismoStruct (2010b), y descripto también en el Informe de Verificación de ese software.

Si bien este modelo está basado en Pavan (2008), se agrega información específica sobre la simulación en el programa considerado.

\subsection{Geometría}

La geometría desarrollada para el prototipo en los Ejemplos de Verificación del programa mencionado es igual a la que se muestra en la Figura 8, mientras que las alturas de piso y nivel se indican en Tabla 11. 
Tabla 11: Altura de pisos y niveles en [m] para el Modelo II, en SeismoStruct (2013b).

\begin{tabular}{c|c|c|c}
\hline Piso & Altura respecto a la sup. de la mesa & Nivel & Altura de nivel \\
\hline Techo & 14.052 & 4 & 3.525 \\
4to & 10.525 & 3 & 3.525 \\
3ro & 7.000 & 2 & 3.500 \\
2do & 3.500 & 1 & 3.500 \\
\cline { 2 - 3 } 1ro & 0.000 & \multicolumn{2}{|c}{-} \\
\hline
\end{tabular}

Se destacan las diferencias en las alturas de piso y de nivel entre este segundo modelo, y las del prototipo (Tabla 1). Para la evaluación de resultados en la sección 6 este modelo fue modificado corrigiendo las alturas y agregando las bases de hormigón armado.

\subsection{Materiales}

\subsubsection{Hormigón}

El hormigón incluido como ancho colaborante de la sección de las vigas principales, se simuló según el modelo de Mander (con-ma) programado en SeismoStruct (2013a), teniendo en cuenta los parámetros mecánicos dados para el hormigón de la losa en la Tabla 4. Para cada ancho colaborante de losa se consideró hormigón confinado en el núcleo (factor de confinamiento 1.2), y hormigón no confinado en la zona perimetral de recubrimiento (factor de confinamiento 1.0).

Dado que la losa no posee estribos, se considera en este trabajo que no debería establecerse un factor de confinamiento de 1.2; aunque este parámetro seguramente no tendrá influencia en los resultados numéricos de la simulación.

El peso específico establecido para el hormigón fue de $24 \mathrm{kN} / \mathrm{m}^{3}$.

\subsubsection{Acero}

Como modelo constitutivo para el acero de las vigas y columnas, se consideró en SeismoStruct (2013a) un modelo bilineal (stl_bl), considerando como parámetros los dados en la Tabla 8 . El peso específico considerado para el acero fue de $78 \mathrm{kN} / \mathrm{m}^{3}$.

\subsection{Secciones}

\subsubsection{Clase de sección considerada}

La sección considerada en SeismoStruct (2013b) para representar las vigas principales fue la "sección doble T compuesta - cpis", según se muestra en la Figura 10. 


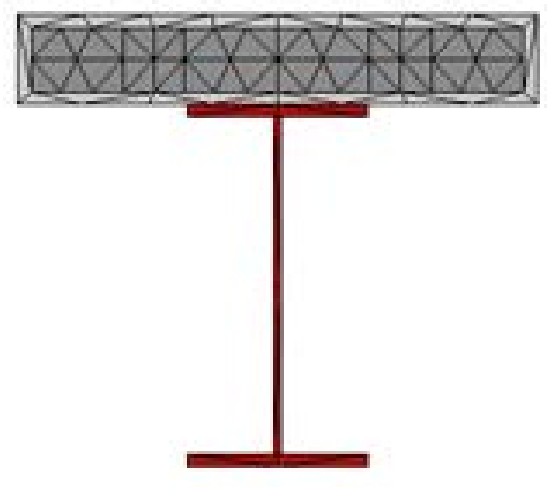

Figura10: Sección doble T compuesta para vigas.

En el caso de las columnas se consideró la sección rectangular hueca (rhs), tal como se muestra en la Figura 11. Estos elementos estructurales no poseían masa agregada.

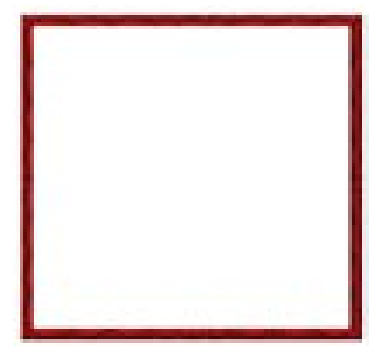

Figura 11: Sección rectangular hueca para columnas.

\subsection{Clases de elementos}

Las vigas y columnas fueron modeladas con elementos inelásticos de pórtico basados en desplazamiento (infrmDB en SeismoStruct, 2013a). Estos elementos 3D viga-columna permiten modelar miembros de pórticos espaciales con no linealidades geométricas y de los materiales (elementos con inelasticidad distribuida).

Para todos los elementos estructurales se hizo una discretización con 100 fibras en cada sección transversal. En SeismoStruct (2013a) se hace uso del llamado "enfoque de fibras" para representar el comportamiento de las secciones transversales, donde cada fibra es asociada a una relación uniaxial esfuerzo-deformación; el estado seccional de esfuerzo-deformación de los elementos viga-columna es obtenido mediante la integración de la respuesta esfuerzo-deformación uniaxial no lineal de las fibras individuales en las cuales ha sido subdividida la sección. Se tiene así en cuenta la propagación de la inelasticidad a lo largo de la longitud del miembro, y en la altura de la sección. 
Los elementos de pórtico con inelasticidad distribuida pueden ser implementados mediante dos formulaciones de elementos finitos: la clásica formulación basada en desplazamientos, y la más reciente formulación basada en fuerzas. Aunque la opción más recomendable es esta última, no se implementó para la simulación del prototipo, ya que se presentaron problemas de convergencia, que impidieron completar el análisis.

\subsection{Vinculos internos y externos}

A nivel de cada losa se consideró un diafragma rígido.

Se consideró empotramiento en todos los nodos de pie de columnas.

\subsection{Consideraciones sobre ajustes en SeismoStruct (2013a)}

Se empleó el método de Skyline como algoritmo de resolución, incluyendo las no linealidades geométricas. Como método de integración de la ecuación de movimiento, fue seleccionado el algoritmo de Hilbert - Hughes - Taylor (con parámetros $\alpha=-0.1, \beta$ $=0.3025 ; \mathrm{y} \gamma=0.6$.

$\mathrm{El}$ amortiguamiento estructural considerado fue proporcional a la rigidez tangente, con parámetro de rigidez de 0.00230775. Este parámetro se calcula con la ec. (1).

$$
\frac{\mathrm{T} \xi}{\pi}=\frac{1.450 .005}{3.14}
$$

Donde $\mathrm{T}$ fue el período fundamental considerado para la estructura, y $\xi$ la relación de amortiguamiento viscoso equivalente. Se destaca el valor bajo considerado para $\square$. Esto se debe a que en la formulación con elementos infrm, se considera la disipación histerética por el comportamiento no lineal del modelo constitutivo elegido para los materiales del elemento, que es la principal fuente de disipación de energía en un análisis dinámico no lineal. El valor de $\xi=0.5 \%$ es para representar la disipación de pequeñas cantidades de energía movilizadas durante la respuesta dinámica, debidas a fenómenos de fricción entre elementos estructurales y no estructurales, y radiación de energía a través de las fundaciones.

La masa adicional, y la excitación en la base para el Modelo II fue igual a la indicada en el Modelo I.

Si en el Modelo II se modifican las alturas de piso según Tabla 1, y se agregan los bloques de hormigón de las bases, se tiene:

i El tiempo computacional para el análisis es de 56 minutos.

ii La suma de reacciones, según análisis estático, es de $1949.76 \mathrm{kN}$, con una diferencia del $5 \%$ respecto del peso total del prototipo dado en Tabla 6.

iii Los períodos para los primeros dos modos se presentan en Tabla 12, los cuales se comparan con los períodos medidos en el prototipo. 
Tabla 12: Períodos del Modelo II vs. período del prototipo en [s].

\begin{tabular}{l|c|c|c}
\hline \multicolumn{1}{c|}{ Direction } & Modelo II & Prototipo & Dif [\%] \\
\hline Longitudinal & 0.82 & 0.76 & 7.9 \\
Transversal & 0.82 & 0.80 & 2.5 \\
\hline
\end{tabular}

\section{Simulación numérica para el modelo III}

Se describe a continuación el modelo desarrollado por los autores de este trabajo, tomando como base los datos del Modelo I.

\subsection{Geometría}

En la Figura 12 se presenta la geometría para este modelo.

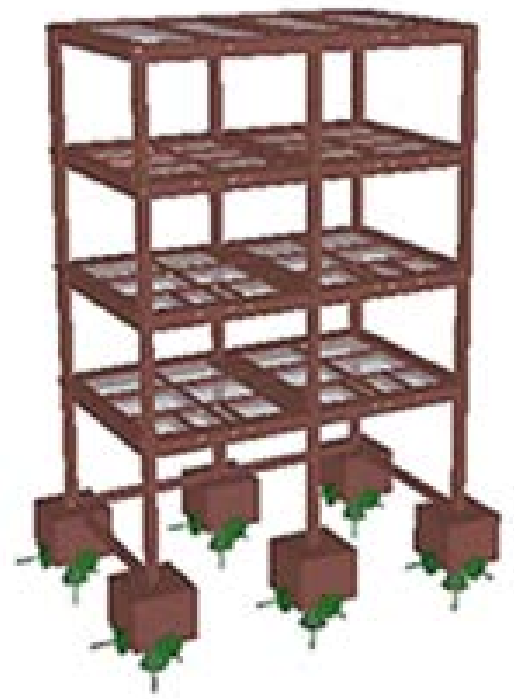

Figura 12: Geometría del Modelo III.

A diferencia del Modelo I de Pavan (2008): i- se consideraron las vigas secundarias; ii- se incluyeron los bloques de hormigón armado que vinculaban el prototipo con la masa vibradora; iii- se distribuyeron las masas por piso según la Tabla 6; y iv- se realizó una actualización del modelo, tomando como variable el amortiguamiento estructural.

Las alturas de piso y de niveles para el modelo en consideración son las dadas en la Tabla 1.

Las bases de hormigón se modelaron como prismas de base cuadrada, con igual momento de inercia que las bases del prototipo. 


\subsection{Materiales}

\subsubsection{Hormigón}

Los parámetros para el hormigón considerado en al ancho colaborante de la sección de las vigas principales fueron los mismos que en Modelo I de Pavan (2008).

Para las bases soporte del pórtico espacial se consideró también el modelo de Mander, con resistencia a compresión $\mathrm{f}_{\mathrm{cu}}=240 \mathrm{MPa}$, resistencia a tracción $\mathrm{f}_{\mathrm{t}}=2.4 \mathrm{MPa}, \mathrm{y}$ deformación para el valor máximo de tensión en el hormigón $\varepsilon_{\mathrm{c}}=0.002$.

Se estableció un peso específico nulo para el hormigón, ya que en las vigas principales de cada nivel, se consideró como masa adicional el peso relativo de todas las cargas relacionadas con ese nivel.

\subsubsection{Acero}

Para las columnas y vigas principales se adoptaron los mismos modelos constitutivos y parámetros que en el Modelo I de Pavan (2008). Mientras que para las vigas secundarias se mantuvo el modelo bilineal, con $\mathrm{E}=200000 \mathrm{MPa}, \mathrm{f}_{\mathrm{y}}=330.0 \mathrm{MPa}$; $\mathrm{y}$ un parámetro de endurecimiento por deformación igual a 0.05 .

Por igual consideración que en el caso del hormigón, no se incluyó el peso específico del acero.

\subsection{Secciones}

\subsubsection{Clase de sección considerada}

Para las columnas y vigas principales se adoptaron las mismas secciones transversales que en el Modelo I de Pavan (2008); y para las vigas secundarias se adoptaron secciones doble T simétricas (denominadas sits en SeismoStruct, 2013a).

\subsubsection{Masa adicional}

Para determinar las masas adicionales en cada viga principal se consideraron los datos de Tabla 5 y 6 , más el esquema de área tributaria de Figura 9. Se llegó, en base a esos datos, a los valores dados en Tabla 13. 
Tabla 13: Masa adicionales agregadas en vigas en [t / m], y pesos por piso en [kN], para el Modelo III.

\begin{tabular}{c|c|c|c}
\hline Piso & Elemento & $\begin{array}{c}\text { Masa adicional } \\
{[\mathrm{t} / \mathrm{m}]}\end{array}$ & $\begin{array}{c}\text { Peso del piso } \\
{[\mathrm{kN}]}\end{array}$ \\
\hline \multirow{3}{*}{5} & $\mathrm{RG} 1$ & 1.3418 & \\
& $\mathrm{RG} 11$ & 1.5650 & 631.72 \\
& $\mathrm{RG} 12$ & 3.1300 & \\
4 & $4 \mathrm{G} 1$ & 1.0125 & 476.65 \\
& $4 \mathrm{G} 11$ & 1.1819 & \\
& $4 \mathrm{G} 12$ & 2.3627 & 473.17 \\
& $3 \mathrm{G} 1$ & 1.0050 & \\
& $3 \mathrm{G} 11$ & 1.1722 & 412.29 \\
& $3 \mathrm{G} 12$ & 2.3444 & \\
\hline \multirow{3}{*}{2} & $2 \mathrm{G} 1$ & 1.0082 & 1993.82 \\
\hline
\end{tabular}

Respecto de la Tabla 6, donde se indicaron los pesos del prototipo, se observa que se tienen los mismos pesos totales por piso, excepto en el segundo nivel. En este caso, como se considera la masa concentrada por piso, no está agregada la masa correspondiente al medio nivel inferior de este piso. Del análisis estático en SeismoStruct (2013a) se obtiene la suma de reacciones verticales igual a $2113.85 \mathrm{kN}$, que incluye la suma de todo los pesos del modelo (donde están consideradas también las bases).

Del análisis de auto-valores en el programa mencionado, se obtuvieron los valores de los períodos indicados en Tabla 14, que se comparan con los períodos medidos en el prototipo.

Tabla 14: Períodos en [s] del segundo modelo vs. período del prototipo.

\begin{tabular}{l|c|c|c}
\hline \multicolumn{1}{c|}{ Direction } & Modelo III & Prototipo & Dif [\%] \\
\hline Longitudinal & 0.82 & 0.76 & 7.9 \\
Transversal & 0.84 & 0.80 & 5.0 \\
\hline
\end{tabular}

\subsection{Clases de elementos, vínculos internos y externos, y excitación en la base}

La selección de elementos, vínculos internos y externos, y la excitación en la base fue igual a la adoptada para el Modelo I de Pavan (2008). 


\subsection{Consideraciones sobre ajustes en SeismoStruct (2013a)}

Como en el Modelo II en SeismoStruct (2013b), se empleó el método de Skyline como algoritmo de resolución, incluyendo las no linealidades geométricas. Como método de integración de la ecuación de movimiento también fue seleccionado el algoritmo de Hilbert - Hughes - Taylor ( $\operatorname{con} \alpha=-0.1, \beta=0.3025 ; \gamma=0.6$ ).

$\mathrm{El}$ amortiguamiento estructural considerado fue proporcional a la rigidez tangente como en el caso del Modelo II en SeismoStruct (2013b), pero calculado en base a una relación de amortiguamiento viscoso equivalente del $2.2 \%$. Se llegó a este valor luego de un proceso iterativo, destinado a mejorar la exactitud de la respuesta numérica.

\section{Estudio de resultados}

\subsection{Comparación entre resultados numéricos y experimentales}

Durante el ensayo solo se midieron desplazamientos y aceleraciones absolutas. Por ello, en esta sección se comparan valores numéricos y experimentales solo para los parámetros mencionados.

Así, se presenta en la Figura 13 la comparación de valores numéricos y experimentales, referido a los valores máximos de desplazamientos relativos a la base, para cada piso. Luego en la Figura 14 se comparan valores numéricos y experimentales correspondientes a las aceleraciones máximas absolutas por nivel. En ambas Figuras los valores numéricos considerados son los del Modelo I y III, es decir el modelo descripto en Pavan (2008) y el desarrollado por los autores de este trabajo.

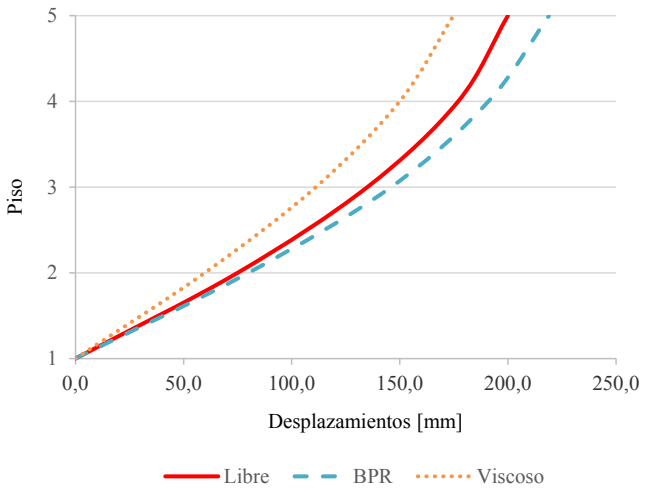

a) Acelerograma dirección $X$

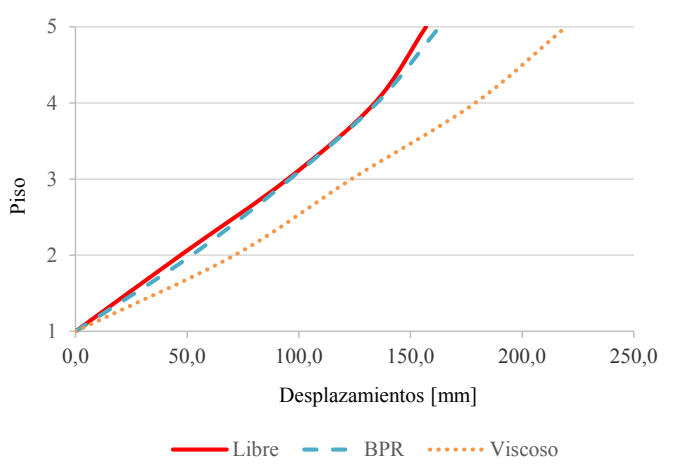

b) Acelerograma dirección $Y$

Figura 13: Valores máximos de desplazamientos relativos a la base. 


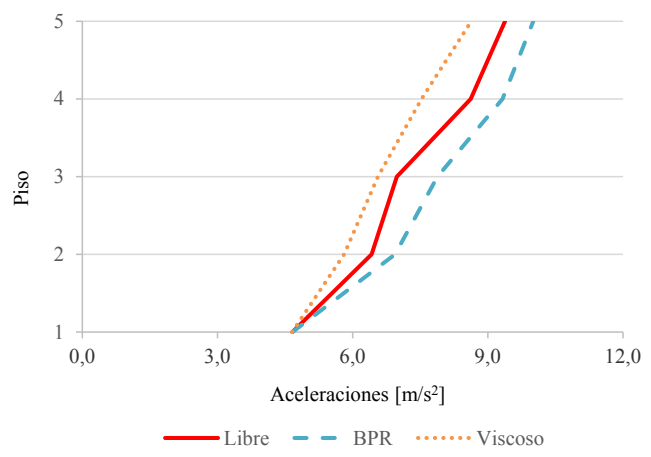

a) Acelerograma dirección $X$

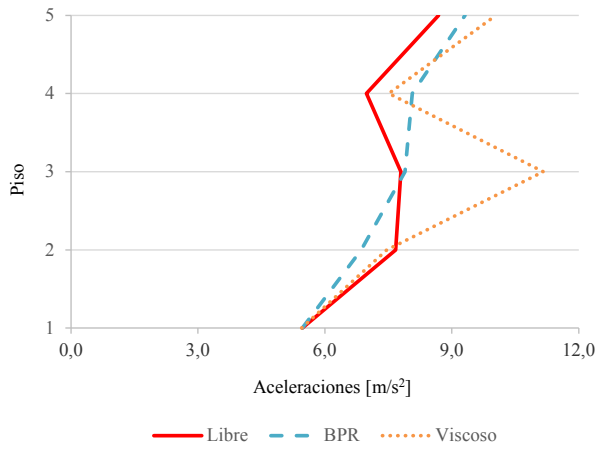

b) Acelerograma dirección $Y$

Figura 14: Valores máximos de aceleraciones absolutas.

Se destaca que el Modelo I de Pavan (2008) aproxima mejor a los resultados experimentales.

En la Tabla 16 se muestran los errores máximos alcanzados en los 3 modelos numéricos considerados. Se agregan entonces los resultados del Modelo II, que es el modelo de Pavan (2008) incorporado en los ejemplos de verificación de SeismoStruct (2013b).

Tabla 15: Errores máximos en [\%] de los valores numéricos respecto los experimentales.

\begin{tabular}{l|c|c|c}
\hline \multicolumn{1}{c|}{ Parámetro } & Modelo I & Modelo II & Modelo III \\
\hline Desplaz. en X & 8.0 & 12.0 & 21.0 \\
Desplaz. en Y & 11.0 & 53.0 & 38.0 \\
Acel. en X & 13.0 & 46.0 & 18.0 \\
Acel. en Y & 16.0 & 27.0 & 17.0 \\
\hline
\end{tabular}

Como antes se indicó, el Modelo I de Pavan (2008) aproxima mejor a los valores experimentales, mientras que el Modelo II (modelo de Pavan, 2008 en SeismoStruct, 2013b) y el Modelo III (desarrollo propio), presentan errores máximos similares.

\subsection{Comparación de historias de las respuestas en los Modelo II y III}

En la Figura 15 se comparan, las historias de desplazamientos relativos a la base, para el techo de los Modelos II y III. Luego en la Figura 16 se comparan las historias de las aceleraciones absolutas para ambos modelos, también en el último nivel. No se considera en esta parte el Modelo I, ya que solo se tienen en Pavan (2008) los valores máximos de respuesta. 


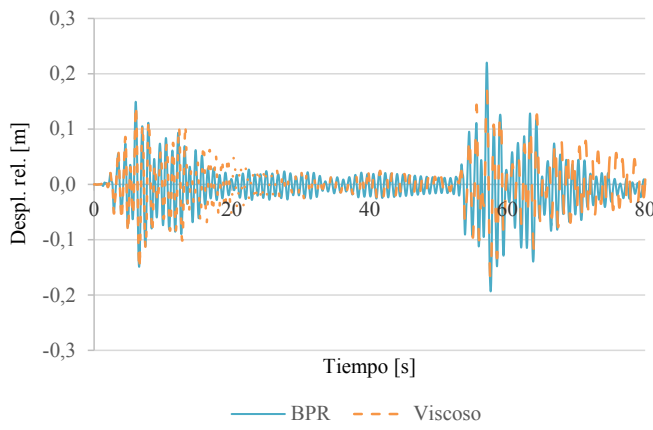

a) Acelerograma dirección $X$

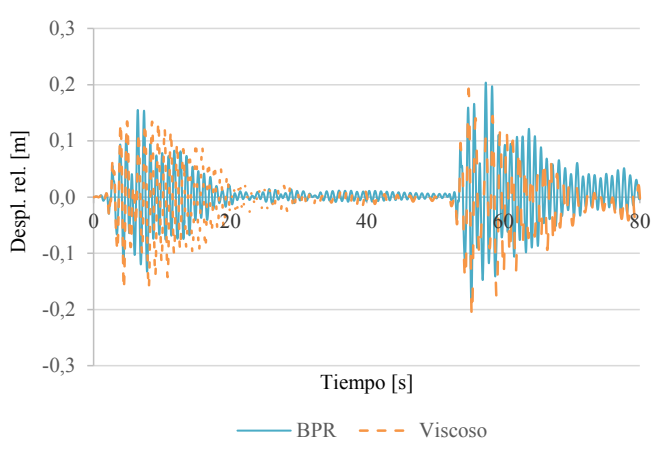

b) Acelerograma dirección $Y$

Figura 15: Historia de los desplazamientos relativos en el techo de Modelos II y III.

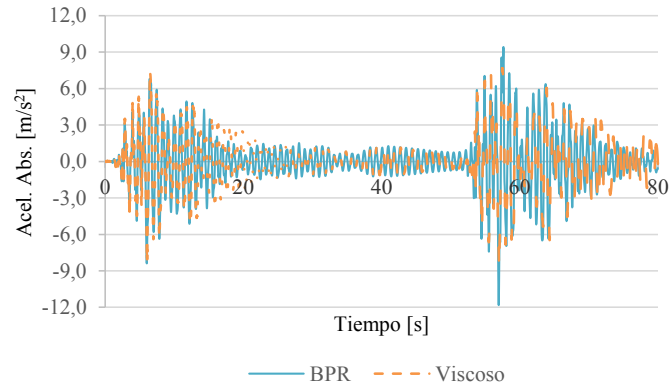

a) Acelerograma dirección $X$

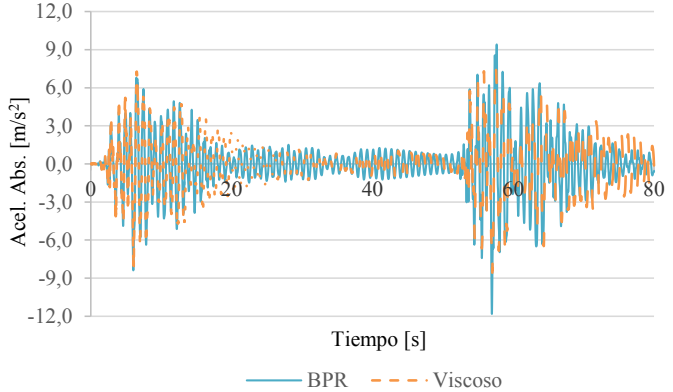

b) Acelerograma dirección $Y$

Figura 16: Historia de las aceleraciones absolutas en el techo de Modelos II y III.

En la Tabla 17 se comparan las dos historias, a través de la raíz cuadrada media RMS (raíz cuadrada de la suma de las diferencias de ordenadas elevadas al cuadrado).

Tabla 16: RMS para valores numéricos de Modelo II y III, según historias de respuesta en el último nivel.

\begin{tabular}{c|c}
\hline Parámetro & RMS \\
\hline Despl. X & 2.4 \\
Despl. Y & 6.5 \\
Acel. X & 128.4 \\
Ace. Y & 247.9 \\
\hline
\end{tabular}

Las gráficas de las historias de desplazamientos y de aceleraciones en la Figura 15 y 16 son aparentemente coincidentes, aunque el valor de RMS indica una mayor diferencia para las historias de aceleraciones. 


\section{Conclusiones}

Este trabajo evalúa el grado de aproximación que puede lograrse en la simulación numérica de una estructura metálica espacial, sometida en una mesa vibradora a las 3 componentes de un acelerograma registrado durante el terremoto de Kobe de 1995. Estas simulaciones se realizaron en el programa de elementos finitos SeismoStruct (2013a), que permite considerar grandes desplazamientos y no linealidad generalizada en los elementos estructurales.

De la estructura en consideración se tuvieron como datos: los parámetros geométricos, los pesos, las leyes constitutivas de los materiales empleados en la estructura, detalle de uniones, especificaciones para los elementos no estructurales, y la excitación medida como input en la base de la mesa. Estos datos fueron proporcionados por un comité organizador de un concurso ciego propuesto antes del ensayo del prototipo.

Para las simulaciones numéricas se emplearon tres modelos numéricos similares, los cuales se basaron en los datos aportados por Pavan (2008). Éstos consistieron en simulaciones simples de la estructura real; donde no se consideraron los elementos no estructurales, ni los detalles de diseño especial para las conexiones viga - columna y columna - base.

Los datos y resultados (valores máximos de desplazamientos y aceleraciones) del primer modelo se tomaron directamente de Pavan (2008). El segundo modelo, también basado en Pavan (2008), está incluido en los Ejemplos de Verificación del programa SeismoStruct (2010b). Pero para este trabajo fue modificado a los efectos de considerar las cotas reales del prototipo, y la existencia de las bases de hormigón armado. Este modelo presentó diferencias respecto a la descripción de Pavan (2008), ya que los valores máximos de desplazamiento y aceleración no son coincidentes a los indicados en la última referencia mencionada. El último modelo, desarrollado por los autores de este trabajo, difiere principalmente de Pavan (2008) en la consideración de las vigas secundarias, y en la determinación del amortiguamiento estructural mediante una actualización del modelo según valores experimentales de frecuencias fundamentales.

Al comparar resultados numéricos y experimentales, referidos a valores máximos de desplazamiento relativo a la base y aceleraciones absolutas por piso, se observó un mejor grado de aproximación para el primer modelo. Los otros dos modelos presentaron un nivel de aproximación similar.

Al comparar las curvas de historias de respuesta del segundo modelo respecto al tercero, se obtuvieron valores bajos de RMS para el caso de los desplazamientos, y valores más altos en el caso de las aceleraciones.

Se estima que para que un modelo numérico pueda reproducir el comportamiento experimental dinámico de una estructura metálica en campo no lineal, debe prestarse especial atención a: influencia de los elementos no estructurales, simulación de las losas, parámetros mecánicos de los materiales que conforman la estructura, y modelos constitutivos para comportamiento no lineal y con grandes desplazamientos. Pero 
principalmente debe establecerse un criterio racional para definir el amortiguamiento estructural global a considerar en la simulación.

\section{References}

Ohsaki, M., Kasai, K., Thiagarajan, G., Yang, Y. and Komiya, Y. (2008).3-D analysis methods for 2007 blind analysis contest.14 ${ }^{\text {th }}$ World Conference on Earthquake Engineering, Beijing, China.

Pavan A. (2008). Blind prediction of a full scale 3D steel frame tested under dynamic conditions. Msc Dissertation, ROSE Shool, Pavia, Italia.

SeismoStruct (2013a), v.6, Seismosoft. A computer program for static and dynamic nolinear analysis of framed structures, online, available from URL: http//www.seismosoft.com.

Seismosoft (2013b). SeismoStruct v6 - Verification Report, disponible en www.seismosoft.com.

Tada M, Ohsaki M, Yamada S, Motoyui S, Kasai K. (2007). E-Defense Test on Full-Scale SteelBuildings: Part 3- Analytical Simulation of Collapse, NEES/E-Defense collaborative researchprogram on steel structures.

Yamada, S.; Suita, K.; Tada; K., Kasai, K; Matsuoka, Y., y Shimada; Y. (2004). Collapse experiment on 4-story steel moment frame: Part 1 outline of test results. Proceedings of the $14^{\text {th }}$ World Conference on Earthquake Engineering, Beijing, China. 


\section{Numerical simulation of a steel structure tested on a shaking table}

\section{SUMMARY}

In the E -Defense laboratory (Japan), a prototype full scale building structure was tested during 2007. That prototype was tested on a shaking table under different levels of the three components of the Takatori record (Kobe earthquake of 1995). The objective of this study is to simulate the experiments with nonlinear computational models. The prototype tested and the models considered were presented. Comparisons between numerical and experimental maximum values and time histories are presented. The characteristics of each model are highlighted, and the considerations in the numerical model to improve structural response predictions are described.

Keywords: earthquake-resistant steel structure, nonlinear dynamic analysis, comparison between numerical and experimental results.

\section{Conclusions}

The accuracy of the numerical simulation of the dynamic behavior of a spatial steel structure; is assessed in this paper. The structure was tested in a shaking table during 2007 under different levels of the three components of the Takatori record (Kobe earthquake of 1995). The software package SeismoStruct is used for the numerical simulations. The information about the experiments was provided by the organizers of a blind exercise.

Three similar numerical models based on published research have been used in this work. The non-structural elements and the details of the beam-column and base-column connections have not been considered.

The data and the results for the first model is based completely on the research mentioned. The second model was taken from the examples of the software used, but some modifications were introduced. And the third model was developed for the authors of this paper. In this last model the following modifications were considered: (i) the secondary beams were simulated, and (ii) the structural damping was obtained by an updating procedure, based in modal parameter measured.

The accuracy of these models were similar.

The conclusions highlight the importance of considering the following aspects in the numerical simulations: (i) the influence of nonstructural elements and slabs; (ii) reliable mechanical properties and constitutive laws of structural materials; and mainly (iii) a rational criterion to define the structural damping. 